\title{
Fermentation Characteristics along the Gastrointestinal Tract after Feeding of Jerusalem Artichoke Meal to Adult Healthy Warmblood Horses
}

\section{Glatter ${ }^{1}$, K. Wiedner ${ }^{2}$, F. Hirche ${ }^{3}$, N. Mielenz ${ }^{4}$, D. Hillegeist ${ }^{5}$, M. Bochnia ${ }^{1}$, A. Cehak ${ }^{7}$, M. Bachmann ${ }^{1}$, J. M. Greef ${ }^{5}$, B. Glaser ${ }^{2}$, P. Wolf ${ }^{6}$, G. Breves ${ }^{7}$ and A. Zeyner ${ }^{1}$}

${ }^{1}$ Group Animal Nutrition, Institute of Agricultural and Nutritional Sciences, Martin Luther University Halle-Wittenberg, Germany

${ }^{2}$ Group Soil Biogeochemistry, Martin Luther University Halle-Wittenberg, Germany

${ }^{3}$ Group Human Nutrition, Martin Luther University Halle-Wittenberg, Germany

${ }^{4}$ Biometrics and Informatics in Agriculture Group, Martin Luther University Halle-Wittenberg, Germany

5 Julius Kuehn Institute, Federal Research Center for Cultivated Plants, Crop and Soil Science, Braunschweig, Germany

${ }^{6}$ University of Rostock, Chair of Nutrition Physiology and Animal Nutrition, Rostock, Germany

${ }^{7}$ University of Veterinary Medicine Hannover, Foundation, Department of Physiology, Hannover, Germany

Corresponding author: M. Glatter, Group Animal Nutrition, Institute of Agricultural and Nutritional Sciences, Martin Luther University HalleWittenberg, 06120 Halle (Saale), Germany, Tel no: 0345-5522679 E-mail: maren.glatter@landw.uni-halle.de

Rec date: March 28, 2016; Acc date: May 24, 2016; Pub date: May 30, 2016

Copyright: (C) 2016 Glatter M, et al. This is an open access article distributed under the terms of the Creative Commons Attribution License, which permits unrestricted use, distribution, and reproduction in any medium, provided the original author and source are credited.

\section{Abstract}

Objective: Prebiotics are used to support the gastrointestinal health via stimulating particular beneficial parts of the autochthonous microflora and enhancing their metabolism. Horses often suffer from gastrointestinal disturbances after feed changes or behavioral stress in response to transport. Therefore, the supplementation with prebiotic compounds might reduce the risk for intestinal dysregulation. The aim of this study was to investigate the influence of supplementation with Jerusalem artichoke meal (JAM) in a recommended prebiotic dosage on fermentation characteristics in the equine gastrointestinal tract.

Methods: Twelve adult healthy horses received crushed oat grains ( $1.2 \mathrm{~g} \mathrm{starch} / \mathrm{kg} \mathrm{BW} \mathrm{x} \mathrm{d}^{-1}$ ) and meadow hay (as

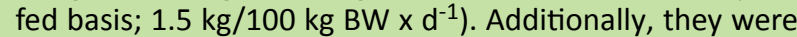
fed either an apparently prebiotic quantity of fructo-

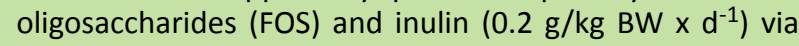
Jerusalem artichoke meal (JAM) or an equal amount of maize cob meal without grains as control (CON) over 3 weeks. On d21, horses were euthanized, gastrointestinal contents were removed from 7 different regions of the gastrointestinal tract, the dry matter (DM) content, $\mathrm{pH}$ value and concentrations of short chain fatty acids (SCFA), $\mathrm{L}$ - and D-lactate and ammonia were measured.

Results: JAM did not had a significant $(P<0.05)$ effect on any of the measured fermentation products and the $\mathrm{pH}$ value as well. Numerically, JAM increased the concentrations of SCFA ( $P>0.05$ ), lactate (both isomers, $P$ $>0.05)$ and ammonia $(P<0.05)$ predominantly in the stomach but had no impact on the $\mathrm{pH}$ value overall. In the hindgut, the stimulation of the microbial activity was limited to the ventral colon only indicated by slightly higher SCFA $(P>0.05)$ and ammonia $(P>0.05)$ but lower L-lactate $(P>0.05)$ concentrations compared to control.
Conclusion: FOS and inulin from JAM seem extensively be fermented already in the stomach of horses. The resulting organic acids might elevate the risk for gastric ulcers. Recently the gastric $\mathrm{pH}$ was buffered by concomitantly elevated ammonia, which requires a careful delineation of the influences of either individual acids or low $\mathrm{pH}$ levels or both together on the mucosa health in both distinct parts of the equine stomach. In the hindgut, the effect of JAM on the microbial activity seems to be much less pronounced than expected or advertised.

Keywords: Gastrointestinal tract; Horse; Microbial fermentation; Prebiotic; Jerusalem artichoke

\section{Introduction}

The equine digestive tract is prone to health disturbances, predominantly after feed changes and overload with easily fermentable carbohydrates [1,2]. This is usually accompanied by alterations in the diversity and activity of the intestinal microbiota $[3,4]$. Prebiotics are supposed to have the potency to promote the guts' health by providing substrates selectively for particular beneficial indigenous microorganisms. In horse feeding, inulin-type fructans with different chain length such as short chain fructo-oligosaccharides (scFOS) or inulin itself are implemented as prebiotic substances. Depending on the amount of intake, both inulin- and phlein-type fructans (the latter deriving from vegetative parts of temperate grasses) have either critical or beneficial properties [2,5-8]. Moreover, the quantity of fermentation products such as short chain fatty acids (SCFA) and lactate varies with the dosage and the composition of the supplemental feed [9]. Feeding scFOS in low $(8 \mathrm{~g} / \mathrm{d})$ or high $(24 \mathrm{~g} / \mathrm{d})$ amounts increases the content of total and individual SCFA in the feces of yearling horses [10]. Contrary, the addition of $30 \mathrm{~g} \mathrm{scFOS/d}$ to a diet for adult horses failed to influence the concentration of SCFA in both 
caecum and colon content [11]. Except for these studies, there is a lack of knowledge dealing with the impact of prebiotics on fermentative parameters in the entire gastrointestinal tract. The passed down assumption, however, that prebiotics will exclusively be fermented in the hindgut [12] might not be transferred to horse nutrition. Fermentation gasses in the breath following inulin ingestion indicate that microbial fermentation already starts in the lower tract [13].

From this we hypothesized that following apparently prebiotic intakes of inulin-type fructans by horses i) fermentative degradation starts already in the stomach and ii) due to this disappearance of prebiotic substances the impact of the microbiota in the hindgut is comparatively low. As indicated by the literature [14] we iii) further hypothesized the concentration of $n$-butyrate will be elevated in a particular degree.

Thus, the aim of the study was to investigate the microbial metabolic responses following apparently prebiotic doses of FOS and inulin originating from Jerusalem artichoke meal to adult healthy warmblood horses in different parts of the gastrointestinal tract.

\section{Materials and Methods}

The experimental procedures were approved by the Animal Welfare Commissioner of the University of Veterinary Medicine Hannover in accordance with the German Animal Welfare Law.

\section{Animals, diets and experimental protocol}

Twelve adult, healthy warmblood horses (10 mares, 1 stallion, 1 gelding) were included in the study. The mean bodyweight (BW) and age were $534 \pm 64.5 \mathrm{~kg}$ and $14 \pm 7.5$ years, respectively. The horses were maintained in individual boxes bedded with wood shavings and had free access to tap water as well as to a salt block.

During a 3 weeks adaptation period, they received crushed oat grains (1.2 $\mathrm{g}$ starch/ $\mathrm{kg} \mathrm{BW} \mathrm{x} \mathrm{d}^{-1}$ ) and meadow hay (as fed basis; $1.5 \mathrm{~kg} / 100 \mathrm{~kg} \mathrm{BW} \times \mathrm{d}^{-1}$ ) in quantities to meet their energy requirements for maintenance [15]. The horses were randomly allocated to either the treatment group (JAM) or the control group (CON).

The treatment group received additionally inulin-type fructans in a doses currently supposed prebiotic [8] (0.2 g FOS + inulin/kg BW $\mathrm{x} \mathrm{d}^{-1}$ ) via Jerusalem artichoke meal (LIVEN $\mathrm{GmbH}$, Zossen, Germany), and the control group alternatively an equivalent amount of maize cob meal without grains.

The dose of prebiotic active substances (fructans including inulin) was calculated according to what was declared for the commercially available JAM. From that, the content of watersoluble carbohydrates was as follows (as fed basis): glucose $1.1 \%$, fructose $5.8 \%$, sucrose $11.5 \%$, fructans $62.7 \%$ (containing $6.3 \%$ inulin in absolute terms). Horses were offered the diet in two equal meals per day (09:00 $\mathrm{h}$ and 15:00 $\mathrm{h}$ ). Because both the Jerusalem artichoke meal and the placebo had the consistency of finely ground material the entire concentrate was moistened to bind fine particles better to coarser ones.

Furthermore, selective feed intake and aspiration was avoided as well. Subsamples of all feedstuffs were taken throughout the entire study for later analyses. The analyzed chemical composition of all feedstuffs is given in Table 1.

Table 1: Analyzed chemical composition of the feedstuffs and calculated contents of metabolizable energy and pre-caecal digestible crude protein and selected amino acids.

\begin{tabular}{|c|c|c|c|c|}
\hline Item & oat & hay & placebo $^{1}$ & prebiotic $^{2}$ \\
\hline dry matter (DM), g/kg & 912 & 944 & 927 & 939 \\
\hline crude ash, g/kg DM & 29 & 52 & 26 & 136 \\
\hline crude protein, g/kg DM & 124 & 90 & 36 & 63 \\
\hline acid ether extract, g/kg DM & 49 & 9 & 8 & 6 \\
\hline crude fiber, g/kg DM & 124 & 349 & 343 & 14 \\
\hline NDF, g/kg DM & 316 & 651 & 799 & 3 \\
\hline ADF, g/kg DM & 178 & 391 & 415 & 11 \\
\hline ADL, g/kg DM & 31 & 47 & 39 & 4 \\
\hline glucose, g/kg DM & 0 & 30 & 9 & 14 \\
\hline fructose, $\mathrm{g} / \mathrm{kg} \mathrm{DM}$ & 0 & 39 & 10 & 63 \\
\hline saccharose, g/kg DM & 11 & 6 & 16 & 122 \\
\hline fructan, $\mathrm{g} / \mathrm{kg} \mathrm{DM}$ & 0 & 40 & 14 & 466 \\
\hline starch, g/kg DM & 498 & 0 & 0 & 0 \\
\hline
\end{tabular}




\begin{tabular}{|c|c|c|c|c|}
\hline $\mathrm{ME}^{3}, \mathrm{MJ} / \mathrm{kg} \mathrm{DM}$ & 12.4 & 6.6 & 7.5 & 11.9 \\
\hline $\mathrm{pcdCP}^{4}, \mathrm{~g} / \mathrm{kg} \mathrm{DM}$ & 75 & 19 & 13 & 56 \\
\hline pcd (lysine) $)^{4}, \mathrm{~g} / \mathrm{kg} \mathrm{DM}$ & 2.92 & 0.80 & 0.05 & 0.36 \\
\hline pcd (cysteine + methionine $)^{4}, \mathrm{~g} / \mathrm{kg} \mathrm{DM}$ & 3.41 & 0.56 & 0.06 & 0.20 \\
\hline pcd (threonine) $)^{4}, \mathrm{~g} / \mathrm{kg} \mathrm{DM}$ & 3.99 & 0.82 & 0.06 & 0.50 \\
\hline
\end{tabular}

${ }^{1}$ maize cob meal without grains; ${ }^{2}$ Jerusalem artichoke meal; ${ }^{3}$ calculation according to [15] and [24]; ${ }^{4}$ calculation according to [15] and [25]

\section{Digesta sampling and preparation}

On d21 of the adaptation period, the horses were euthanized by a veterinarian. Horses were euthanized approximately 1 hour after they received the half of the daily concentrate ration. This served thereto to assure that the foregut (especially the stomach) contains a sufficient quantity of digesta for sampling. The horses were sedated with romifidine $(0.12 \mathrm{mg} / \mathrm{kg} \mathrm{BW})$. The anesthesia was induced with a combination of diazepam $(0.05 \mathrm{mg} / \mathrm{kg}$ BW) and ketamine $(2.2 \mathrm{mg} / \mathrm{kg} \mathrm{BW})$. Finally the horses were euthanized with pentobarbital $(60 \mathrm{mg} / \mathrm{kg} \mathrm{BW})$. Digesta samples were collected immediately after the death from 7 different regions of the gastrointestinal tract: Stomach (Pars nonglandularis [PN], Pars glandularis $[\mathrm{PG}]$, and mixed stomach content $[\mathrm{MC}])$, small intestine (SI), caecum (CAE), and colon: ventrale (CV), dorsale (CD) as well as transversum (CT)). Because of its extremely watery consistency, small intestinal content was used as native material. Digesta samples from the other parts of the digestive tract were prepared for $\mathrm{pH}$ measurement and further analyses as described formerly for equine faeces samples [16]. For this, 50 grams of digesta were mixed with $50 \mathrm{~mL}$ distilled water immediately post mortem. The suspension was covered with aluminum foil to avoid interchange of gases and incubated for $60 \mathrm{~min}$. After that, the $\mathrm{pH}$ value was measured, the suspension centrifuged $(4,000 \times \mathrm{g})$ for $10 \mathrm{~min}$ at room temperature (RT), the supernatant collected in plastic tubes $(2 \mathrm{~mL})$ and stored at $-20^{\circ} \mathrm{C}$ until analysis of organic acids and ammonia. Samples from the particularly watery digesta from the small intestine was centrifuged and stored without any previous dilution.

\section{Analyses}

\section{Feedstuffs and digesta}

The feedstuffs were analyzed for dry matter (DM), proximate nutrients including starch and amino acids according to official methods in which for starch the enzymatic procedure was applied $[17,18]$.

In the digesta, the DM content only was determined with the same method as for feedstuffs. The method from Pavis et al. [16] modified by Hillegeist and Greef was used for detection of water-soluble carbohydrate contents including fructans in the feedstuffs, and the characterization of the structure of fructan isomers as separation according to the degree of polymerization (DP).

\section{$\mathrm{pH}$ value}

The $\mathrm{pH}$ value in the digesta-water-mixtures and native digesta (small intestine), respectively, was measured potentiometrically by use of a $\mathrm{pH}$ meter (Mettler - Toledo, Ohio, USA; precision: \pm 0.01 , temperature compensation: $0^{\circ} \mathrm{C}-$ $\left.100^{\circ} \mathrm{C}\right)$.

\section{SCFA}

The liquid phase of digesta samples (supernatant of the digesta-water-mixture or native liquid phase) were analyzed for SCFA concentrations according to the method from Geißler as described by Zeyner et al. [19]. The analysis was performed using a gas chromatograph (Shimadzu 17A GC, Jena, Germany) with a flame ionization detector and a separation column FFAP $30 \mathrm{~mm} \times 0.25 \mathrm{~mm}$ (Supelco, Pennsylvania, USA) but without use of a water cooling sample rack. Helium was taken as carrier gas (3.99 L/h, split 40:1). The temperature program started at an initial temperature of $80^{\circ} \mathrm{C}$ and heated up at a rate of $10^{\circ} \mathrm{C}$ up to $110^{\circ} \mathrm{C}$, then at $15^{\circ} \mathrm{C}$ up to the end temperature of $175^{\circ} \mathrm{C}$. The temperature was held for $4 \mathrm{~min}$. The total cycle time for one measurement was 11:33 min. For analysis, the supernatant of the digesta-water-mixture was thawed at room temperature and centrifuged $(2,000 \times \mathrm{g})$ for 5 min. Five hundred microliter of the supernatant were transferred into a separate tube ( $1.5 \mathrm{~mL}$ Eppendorf tube). The supernatant was mixed with $50 \mu \mathrm{L}$ of the internal standard solution and centrifuged $(2,000 \times \mathrm{g})$ for $5 \mathrm{~min}$ to segregate the protein precipitation. Then the clear solution was transferred into a micro vial and immediately injected into the GC. The concentrations of the individual SCFA (acetic acid, propionic acid, iso-valeric acid, iso-butyric acid, $n$-valeric acid, 3-methylbutyric acid [ $n$-butyric acid], $n$-caproic acid) in the supernatant of the digesta-water-mixture were calculated upon the internal standard.

\section{L- and D-lactate}

L- and D-lactate were analyzed according to the method of Scheijen et al. [20] by using HPLC-MS/MS after sample preparation according to Henry et al. [21]. As the internal standard (IST) sodium DL-lactate-3, 3, 3-d 3 (CDN Isotopes, Quebec, Canada) was taken. First, the samples were thawed at room temperature, mixed and centrifuged for $5 \min (18,000 \times$ g) at $4^{\circ} \mathrm{C}$. Assuming that the foregut contains higher concentrations of lactate, the samples were treated differently according to their origin in the gastrointestinal tract. After centrifugation, the supernatant $(25 \mu \mathrm{L}$ from samples of the stomach and the small intestine; $1000 \mu \mathrm{L}$ from samples 
belonging to the hindgut) were transferred in a separate Eppendorf tube $(1.5 \mathrm{~mL})$. The supernatants of the foregut samples were mixed with $200 \mu \mathrm{L}$ of the IST $(3 \mathrm{~mol} / \mathrm{L}$, respectively) and $825 \mu \mathrm{L}$ distilled water, the supernatants of the hindgut samples were instead mixed with $50 \mu \mathrm{L}$ of the IST $(0.3 \mathrm{mmol} / \mathrm{L}$, respectively). Both types of samples were mixed with $25 \mu \mathrm{L}$ of hydrochloride acid $27 \%(\mathrm{w} / \mathrm{w})$ and then loaded to the Extrelut NT1 columns (Merck, Darmstadt, Germany). The samples were eluted with $6 \mathrm{~mL}$ 2-methyl-2-butanol: chloroform (ratio 11:9), then extracted by $1 \mathrm{~mL}$ ammonium hydroxide $(0.1 \mathrm{~mol} / \mathrm{L}$; solved in water). After centrifugation at $800 \times \mathrm{g}$ for $3 \mathrm{~min}$ the aqueous phase was transferred in a new $2 \mathrm{~mL}$ Eppendorf tube ( $100 \mu \mathrm{L}$ for the foregut samples; and the entire aqueous phase for the hindgut samples) and evaporated at $65^{\circ} \mathrm{C}$ under a stream of nitrogen. After that, the residue was dissolved in $400 \mu \mathrm{L}$ methanol, mixed thoroughly and was then centrifuged for $5 \mathrm{~min}(18,000 \times \mathrm{g})$ at room temperature. The supernatant was transferred to an opaque HPLC vial and evaporated at $50^{\circ} \mathrm{C}$ under a stream of nitrogen. Residues were mixed with $100 \mu \mathrm{L}$ of diacetyl-L-tartric anhydride $(50 \mathrm{mg} / \mathrm{mL}$ in dichloromethane: acetic acid, ratio 4:1) and incubated for 30 $\min$ at $75^{\circ} \mathrm{C}$ in closed vials for derivatization. Subsequently, the vials were opened and the samples were evaporated at $50^{\circ} \mathrm{C}$ under a stream of nitrogen. Conclusively, the residues were dissolved in $100 \mu \mathrm{L}$ acetonitrile: water (ratio 1:2) and transferred in vials with inserts. Samples were measured using an API 2000 (Applied Biosystems, Darmstadt, Germany) in combination with an HPLC 1100 (Agilent, Waldbronn, Germany) using an Hypersil ODS column (150 mm $\times 2 \mathrm{~mm}, 5$ $\mu \mathrm{m}$; VDS optilab, Berlin, Germany) at $25^{\circ} \mathrm{C}$. The temperature of the ESI-source was set to $300^{\circ} \mathrm{C}$. The eluent was $1.5 \mathrm{mmol} / \mathrm{L}$ ammonium formate $(\mathrm{pH} 3.6)$ in water: acetonitrile (99:1) at a flow rate of $150 \mu \mathrm{L} / \mathrm{min}$. After a run of $13 \mathrm{~min}$, the column was washed for $4 \mathrm{~min}$ with acetonitrile at $250 \mu \mathrm{L} / \mathrm{min}$ and reequilibrated. Lactic acids were measured using the mass transition of $305 / 89 \mathrm{~m} / \mathrm{z}$ and the IST at $308 / 89 \mathrm{~m} / \mathrm{z}$.

\section{Ammonia}

Ammonia was analyzed according to Conway [22], modified by Voigt and Steger [23]. Therefore, the samples were thawed at room temperature. Afterwards, $1 \mathrm{~mL}$ of the digesta-water mixture was transferred into a micro-diffusion vessel and incubated at room temperature for $24 \mathrm{~h}$. Ammonia diffused from the sample into the boric acid und changed the indicator from red to green. After the incubation period, hydrochloride acid $(0.01 \mathrm{~mol} / \mathrm{L})$ was used for titration until the indicator reaches the initial color. The amount of ammonia in the sample can be calculated by the depleted amount of hydrochloride acid using the following equitation:

$$
\mathrm{c}_{\mathrm{A}}=\left(\left(\mathrm{V}_{\mathrm{HCL}} \times 0.17\right) / \mathrm{M}_{\mathrm{NH} 3}\right) \times 1,000
$$

where $c_{A}$ is the concentration of ammonia in the sample (in $\mathrm{mmol} / \mathrm{L}$ ), $\mathrm{V}_{\mathrm{HCL}}$ is the volume (in $\mathrm{mL}$ ) of hydrochloride acid, which was used to reach the initial indicator color and $M_{N H 3}$ is the molar mass (in $\mathrm{g} / \mathrm{mol}$ ) of ammonia. Assuming that $1 \mathrm{~mL}$ hydrochloride acid neutralizes $0.17 \mathrm{mg} / \mathrm{mL}$ ammonia, the factor 0.17 is used to relate the depleted volume of hydrochloride acid with the amount of ammonia in the sample.

\section{Calculations}

Basing on the analyzed chemical composition of the feed, the contents of metabolizable energy (ME) and pre-caecal digestible (pcd) crude protein ( $p c d C P$ ), pcd methionine and cysteine (as sum of sulfur-containing amino acids) and pcd threonine were calculated according to Kienzle and Zeyner [24] and Zeyner et al. [25], respectively. With subject to digesta samples, the concentrations of fermentation products (total and individual organic acids, ammonia) in the native liquid phase of the digesta from the respective parts of the digestive tract were calculated basing on the analyzed DM content and the measured concentration of the fermentation product in the supernatant of the digesta-water-mixture using the equation given by Zeyner et al. [19]. For digesta samples from the small intestine this kind of calculation was dispensable because all analyses were performed directly in the liquid digesta phase.

\section{Statistical analyses}

Statistical analysis was performed using SAS (version 9.4, SAS Inst. Inc., Cary, NC, USA). Results were reported as means \pm SD or least square means (LSmeans) \pm SE. Data were analyzed by PROC MIXED (two-way ANOVA with repeated measures) using a model with the fixed factors treatment (CON and JAM), part of the gastrointestinal tract (PN, PG, MC, $\mathrm{SI}, \mathrm{CAE}, \mathrm{CV}, \mathrm{CD}$ and $\mathrm{CT}$ ), and the interaction between treatment and part. The simultaneous analysis including all parts of the gastrointestinal tract was not possible because (a) no or small variation of the traits was observed in some parts (PN, PG, MC or SI), (b) the assumption of normality of the residual effects was rejected following Shapiro-Wilk test, and (c) including of the complete covariance matrix of the eight observations per animal lead to convergence problems on the estimation of the model parameters. Therefore, parts of the digestive tract were clustered in "foregut" (including PN, PG, $\mathrm{MC}$ and $\mathrm{SI}$ ), and "hindgut" (including CAE, CV, CD and CT). In order to take into account the repeated measures, the residual effects corresponding to the four observations of one animal within "foregut" and "hindgut" were considered as dependent with residual covariance matrix $R$. The following competing covariance structures of $R$ were tested using the repeated statement of the MIXED procedure: unstructured (type $=U N$ ), exchangeable (type $=$ CSH), Toeplitz (type = TOEPH (4), and autoregressive (type $=\mathrm{ARH}(1)$ ). In all structures inhomogeneous residual variances were assumed. The final covariance structure was selected using AIC-values based on the log-likelihood function of the REML-method. Comparison between the different parts of the gastrointestinal tract was made using the post hoc Tukey-Kramer test. The multiple t-test implemented in PROC MIXED (two-way repeated measures ANOVA with Kenward-Roger approximation to the degrees of freedom) was used to compare the two groups (CON and JAM) within the parts of the two tracts ("foregut" and "hindgut"). The Shapiro-Wilk test was applied to check the normality of the studentized residuals. In the case of significant differences and of rejecting the normal distribution, the Wilcoxon rank 
sum test was additionally taken to compare the two groups within the parts. Data were statistical significant at $P<0.05$.

\section{Results}

\section{Chemical composition of the Jerusalem artichoke meal}

The contents of water-soluble carbohydrates determined in the pooled material sampled throughout the entire study was largely different from the declared ones. The analyzed contents were as follows (as fed basis): glucose 1.4\%, fructose $6.3 \%$, sucrose $12.2 \%$, fructans $46.6 \%$ (content of inulin depending from what the DP range is defined being inulin). The fructan of the JAM product was represented by particularly high and largely similar percentages of isomers up to DP 22.

Table 2: Dry matter content (in \%; mean \pm SD) and concentrations (in mmol/L; LSmeans \pm SE) of individual short chain fatty acids (SCFA) along the gastrointestinal tract of 6 control horses (CON) and 6 horses fed with Jerusalem artichoke meal (JAM).

\begin{tabular}{|c|c|c|c|c|c|c|c|c|}
\hline \multicolumn{9}{|c|}{ part of the gastrointestinal tract ${ }^{1}$} \\
\hline Item & PN & PG & MC & SI & CAE & CV & CD & CT \\
\hline \multicolumn{9}{|l|}{ DM } \\
\hline $\mathrm{CON}^{2}$ & $21 \pm 4$ & $19 \pm 4$ & $21 \pm 5$ & $6 \pm 1$ & $7 \pm 2$ & $10 \pm 2$ & $10 \pm 2$ & $16 \pm 4$ \\
\hline $\mathrm{JAM}^{3}$ & $24 \pm 6$ & $20 \pm 3$ & $21 \pm 3$ & $6 \pm 1$ & $8 \pm 2$ & $10 \pm 2$ & $11 \pm 2$ & $13 \pm 5$ \\
\hline \multicolumn{9}{|c|}{ acetic acid } \\
\hline $\mathrm{CON}^{2}$ & $22.7 \pm 4.5$ & $14.7 \pm 5.7$ & $15.7 \pm 2.6$ & $3.9 \pm 1.2$ & $70.4 \pm 7.3$ & $76.7 \pm 7.5$ & $70.4 \pm 8.1$ & $29.7 \pm 4.7$ \\
\hline $\mathrm{JAM}^{3}$ & $30.9 \pm 4.5$ & $22.8 \pm 5.7$ & $24.8 \pm 2.6$ & $2.8 \pm 1.2$ & $54.5 \pm 7.3$ & $84.0 \pm 7.5$ & $70.7 \pm 8.1$ & $35.4 \pm 4.7$ \\
\hline \multicolumn{9}{|c|}{ propionic acid } \\
\hline $\mathrm{CON}^{2}$ & $1.8 \pm 1.2$ & $0.5 \pm 0.3$ & $0.3 \pm 0.4$ & $0.0 \pm 0.1$ & $50.9 \pm 5.9$ & $55.2 \pm 5.6$ & $41.9 \pm 5.0$ & $19.7 \pm 3.2$ \\
\hline $\mathrm{JAM}^{3}$ & $1.4 \pm 1.1$ & $0.3 \pm 0.3$ & $0.7 \pm 0.4$ & $0.1 \pm 0.1$ & $41.5 \pm 5.2$ & $58.2 \pm 5.6$ & $45.4 \pm 5.0$ & $23.7 \pm 3.2$ \\
\hline \multicolumn{9}{|c|}{ iso-butyric acid } \\
\hline $\mathrm{CON}^{2}$ & $0.7 \pm 0.4$ & $0.0 \pm 0.0$ & $0.9 \pm 0.4$ & $0.0 \pm 0.0$ & $2.5 \pm 0.3$ & $9.4 \pm 0.8$ & $8.2 \pm 1.0$ & $3.3 \pm 0.7$ \\
\hline $\mathrm{JAM}^{3}$ & $0.0 \pm 0.0$ & $0.3 \pm 0.4$ & $0.1 \pm 0.4$ & $0.0 \pm 0.0$ & $2.0 \pm 0.3$ & $8.7 \pm 0.8$ & $9.6 \pm 1.0$ & $4.6 \pm 0.7$ \\
\hline \multicolumn{9}{|c|}{$n$-butyric acid } \\
\hline $\mathrm{CON}^{2}$ & $11.5 \pm 8.3$ & $4.9 \pm 2.7$ & $7.6 \pm 4.5$ & $0.3 \pm 0.1$ & $31.3 \pm 3.0$ & $38.1 \pm 4.8$ & $28.9 \pm 5.3$ & $11.1 \pm 2.2$ \\
\hline $\mathrm{JAM}^{3}$ & $28.3 \pm 8.3$ & $8.3 \pm 2.7$ & $18.8 \pm 4.5$ & $0.3 \pm 0.1$ & $25.8 \pm 3.0$ & $49.3 \pm 4.8$ & $27.4 \pm 5.3$ & $11.9 \pm 2.2$ \\
\hline \multicolumn{9}{|c|}{ iso-valeric acid } \\
\hline $\mathrm{CON}^{2}$ & $1.6 \pm 1.0$ & $2.1 \pm 2.5$ & $6.4 \pm 3.4$ & $0.3 \pm 0.2$ & $2.5 \pm 0.4$ & $11.2 \pm 1.4$ & $9.9 \pm 1.2$ & $3.7 \pm 0.8$ \\
\hline $\mathrm{JAM}^{3}$ & $1.5 \pm 1.0$ & $3.4 \pm 2.5$ & $2.1 \pm 3.4$ & $0.1 \pm 0.2$ & $1.8 \pm 0.4$ & $12.1 \pm 1.4$ & $10.6 \pm 1.2$ & $4.3 \pm 0.8$ \\
\hline \multicolumn{9}{|c|}{$n$-valeric acid } \\
\hline $\mathrm{CON}^{2}$ & $0.4 \pm 0.3$ & $0.0 \pm 0.0$ & $0.0 \pm 0.0$ & $0.1 \pm 0.3$ & $2.4 \pm 0.3$ & $4.8 \pm 0.6$ & $4.0 \pm 0.4$ & $1.9 \pm 0.3$ \\
\hline $\mathrm{JAM}^{3}$ & $0.0 \pm 0.0$ & $0.3 \pm 0.3$ & $0.9 \pm 0.3$ & $0.1 \pm 0.3$ & $2.2 \pm 0.3$ & $5.2 \pm 0.6$ & $3.9 \pm 0.4$ & $2.2 \pm 0.3$ \\
\hline \multicolumn{9}{|c|}{$n$-caproic acid } \\
\hline $\mathrm{CON}^{2}$ & $0.0 \pm 1.0$ & $0.0 \pm 0.0$ & $2.8 \pm 1.0$ & $0.0 \pm 0.0$ & $0.0 \pm 0.0$ & $0.0 \pm 0.0$ & $0.0 \pm 0.0$ & $0.0 \pm 0.0$ \\
\hline
\end{tabular}

\section{General observations}

In general, the horses consumed the moistened concentrates quite well and did not exhibit obvious signs of gastrointestinal disturbances. In the stomach of one horse of the placebo group, however, mucosal lesions particularly alongside the margo plicatus were observed post mortem.

\section{Digesta characteristics}

\section{Dry matter}

Irrespective of the diet $(P>0.05)$, the DM content of the digesta (Table 2 ) was highest in the stomach $(\approx 21 \%$ ), particularly low in the small intestine $(\approx 6 \%)$ with further gradual increase until the colon transversum $(\approx 15 \%)$. Along the majority of the digestive tract the digesta DM was numerically higher after feeding of JAM vs. CON, with inverse relation in the colon transversum $(P>0.05)$. 


\begin{tabular}{|c|c|c|c|c|c|c|c|c|}
\hline $\mathrm{JAM}^{3}$ & $0.0 \pm 0.0$ & $0.2 \pm 1.0$ & $1.0 \pm 1.0$ & $0.4 \pm 1.0$ & $0.1 \pm 0.1$ & $0.0 \pm 0.1$ & $0.1 \pm 0.1$ & $0.1 \pm 0.1$ \\
\hline \multicolumn{9}{|c|}{$\begin{array}{l}{ }^{1} \text { part of the gastrointestinal tract: } \mathrm{PN}=\text { Pars nonglandularis; } \mathrm{PG}=\text { Pars glandularis; } \mathrm{MC}=\text { mixed content }(\mathrm{stomach}) ; \mathrm{SI}=\mathrm{small} \text { intestine; } \mathrm{CAE}=\mathrm{caecum} ; \mathrm{CV}=\mathrm{colon} \\
\text { ventrale; } \mathrm{CD}=\text { colon dorsale; } \mathrm{CT}=\text { colon transversum; }{ }^{2} \mathrm{CON}=\text { control group fed with maize cob meal without grains; }{ }^{3} \mathrm{JAM}=\text { treatment group supplemented with } \\
\text { Jerusalem artichoke meal; Statistical analysis did not indicate any significant differences between CON and JAM group horses for a given segment of the digestive } \\
\text { tract }(\mathrm{P}>0.05)\end{array}$} \\
\hline
\end{tabular}

\section{$\mathrm{pH}$ value}

The $\mathrm{pH}$ values did not show any differences between the control and the treatment group (Figure 1; $\mathrm{P}>0.05$ ) in any part of the gastrointestinal tract. In the stomach, the $\mathrm{pH}$ value reached $4.5 \pm 1.1$ on average in the Pars nonglandularis and $3.4 \pm 0.8$ on average in the Pars glandularis (approximately 1 hour postprandial) in both groups. The $\mathrm{pH}$ value in the small intestine was higher than in the stomach with $8.0 \pm 0.1$ (SI) vs. $3.9 \pm 0.7(\mathrm{MC})$, but was similar within both groups. In the further gastrointestinal tract, the $\mathrm{pH}$ value declined from $6.7 \pm$ 0.2 in the caecum to $6.4 \pm 0.3$ in the colon transversum, irrespective of the treatment $(P>0.05)$.

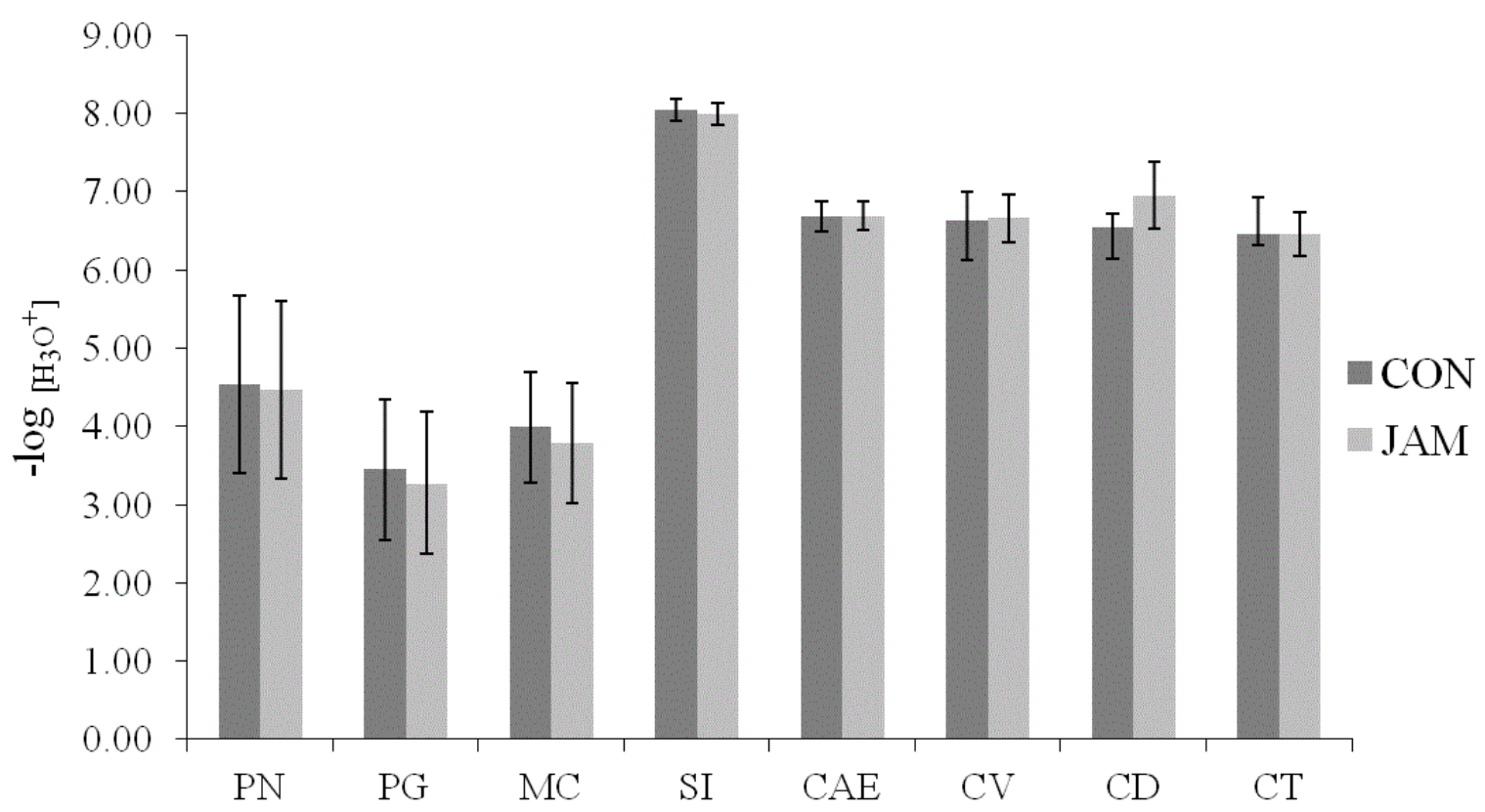

Figure 1: LSmeans $( \pm S E)$ of the $\mathrm{pH}$ value $\left(-\log _{[\mathrm{H3O+}]}\right)$ along the gastrointestinal tract after feeding of JAM $(n=6)$ or CON $(n=6)$.

\section{SCFA}

Irrespective of the diet, concentrations of total SCFA were particularly high in the hindgut and low in the small intestine with the following order along the digestive tract: $C V>C D>$ $\mathrm{CAE}>\mathrm{CT}>\mathrm{PN}>\mathrm{PG}>\mathrm{SI}$. In the non-glandular region of the stomach, the content of total SCFA was about 1.7-fold as high as in the glandular part. In general, the treatment group (JAM) showed numerically higher concentrations of total SCFA in almost all parts of the gastrointestinal tract, except for caecum and small intestine (Table 2; P > 0.05). The difference between diets was particularly high in the non-glandular region of the stomach. Total SCFA in the PN amounted to two-thirds only in CON vs. JAM group horses. The supplemental feeding of JAM resulted in a tendential higher concentration of acetic acid in the mixed content of the stomach $(P>0.05)$ as well as in higher concentrations in the Pars nonglandularis $(P>0.05)$ and Pars glandularis ( $P>0.05$; Table 2). Except for the caecum, the concentration of acetic acid was higher in all parts of the hindgut in the JAM feeding group in comparison to the control group (Table 2; $\mathrm{P}>0.05$ ). The concentration of propionic acid was elevated in the mixed content of the stomach $(P>0.05)$ as well as in the colon (CV, CD and CT; Table 2; P > 0.05) but lower in the Pars nonglandularis, Pars glandularis, small intestine and caecum (Table 2; P > 0.05) with the addition of JAM in the diet. Iso-butyric acid concentration increased only in the Pars glandularis, colon dorsale and colon transversum (Table 2; P > 0.05 ) but was reduced in the other parts of the gastrointestinal tract. The concentration of $n$-butyric acid raised numerically in almost all parts of the digestive tract with exception of the caecum and the dorsal colon (Table 2; P > 0.05). Feeding of JAM resulted in an elevated concentration of iso-valeric acid in the stomach ( $P N$ and $P G)$ as well as in the colon (CV, CD and CT; Table 2; $P>0.05)$. The concentration of the isomer $n$ valeric acid increased in the Pars glandularis, mixed content of the stomach and also in the colon (ventrale and transversum; (Table 2; $\mathrm{P}>0.05$ ). $\mathrm{N}$-caproic acid was measured only in low concentrations and in a few samples, predominantly in the JAM group (Table 2; $P>0.05$ ). The percentage distribution of the SCFA was affected by the addition of JAM in the diet, mainly in the foregut (Figure 2). In the Pars nonglandularis of the JAM group, the percentage of $n$-butyric acid increased with 
coincident reduction of propionic acid but with no impact on the percentage of acetic acid (Figure 2a; $\mathrm{P}>0.05$ ). The same effect was noticed in the Pars glandularis but with a significant lower proportion of propionic acid in the JAM group (Figure $2 b ; P<0.05)$. In the further gastrointestinal tract, the individual SCFA were distributed almost similarly in the control and the treatment group except for the ventral colon. Therein, the percentage of $n$-butyric acid was significant higher in the JAM group compared to the control group (CON: $19.5 \pm 1.66 \%$ JAM: $22.5 \pm 1.37 \%$; $\mathrm{P}<0.05)$ with a slightly coincident reduction of the percentage of acetic acid (CON: $39.1 \pm 3.80 \%$; JAM: $38.8 \pm 2.62 \% ; \mathrm{P}>0.05)$ and propionic acid (CON: $28.2 \pm$ 3.82\%; JAM: $26.8 \pm 1.64 \%$; $P$ > 0.05).
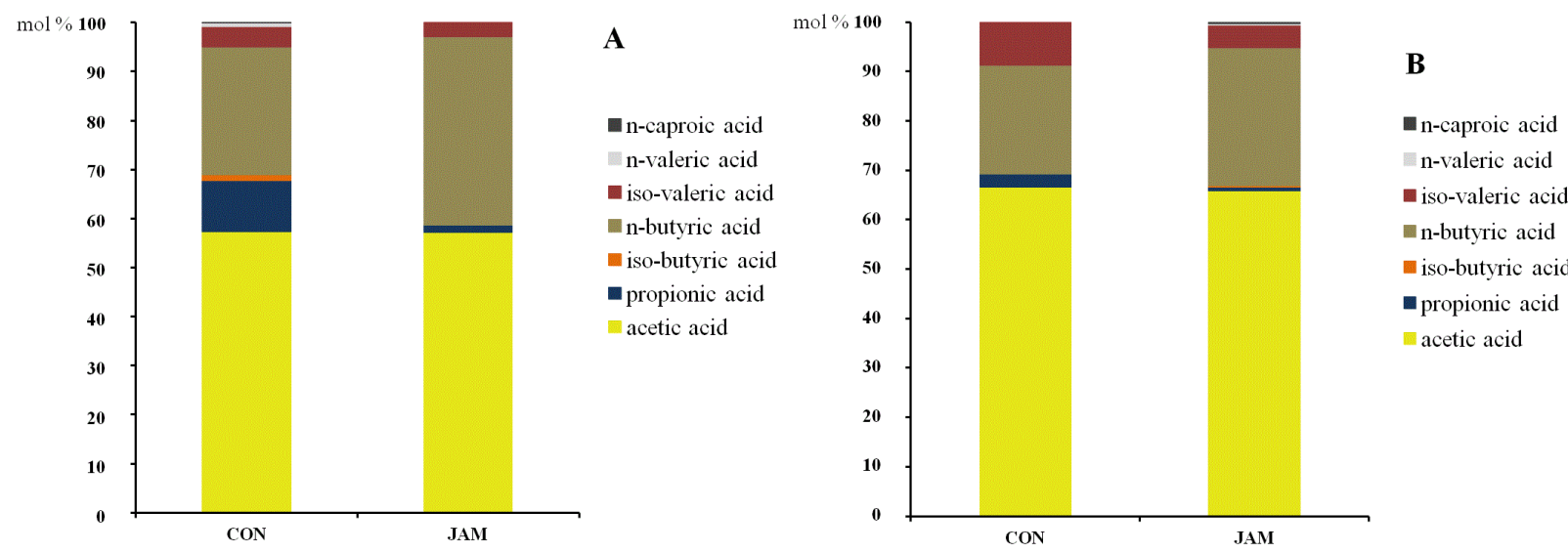

Figure 2: Mean percentage distribution of different short chain fatty acids in the Pars nonglandularis (A) and Pars glandularis (B) of 6 horses fed the control diet (CON) and 6 horses supplemented with a Jerusalem artichoke meal (JAM).

\section{L- and D-lactate}

The concentration of both L- and D-lactate was tendentially higher in the JAM group in both parts of the stomach $(P>$ 0.05). In the small intestine, the concentration of L-lactate was slightly elevated in the CON group compared with the treatment group and vice versa regarding the D-lactate concentration (Table 3; P > 0.05).
Furthermore, the concentration of L-lactate was significantly lower after feeding of JAM in comparison to the control group, exclusively in the ventral colon (Table 3; $\mathrm{P}<0.05$ ).

In the colon transversum, the concentrations of L-lactate as well as D-lactate were tendentially lower in the treatment group (Table 3; $\mathrm{P}>0.05$ ).

Table 3: Concentrations (in mmol/L; LSmeans $\pm \mathrm{SE}$ ) of ammonia and lactate (L-lactate as well as D-lactate) along the gastrointestinal tract of 6 control horses (CON) and 6 horses fed with Jerusalem artichoke meal (JAM).

\begin{tabular}{|c|c|c|c|c|c|c|c|c|}
\hline \multicolumn{9}{|c|}{ part of the gastrointestinal tract ${ }^{1}$} \\
\hline Item & PN & PG & MC & SI & CAE & cV & CD & CT \\
\hline \multicolumn{9}{|c|}{ ammonia } \\
\hline $\mathrm{CON}^{2}$ & $9.7 \pm 1.4$ & $6.5^{\mathrm{a}} \pm 0.5$ & $8.5 \pm 0.7$ & $8.6 \pm 1.3$ & $2.7 \pm 0.6$ & $7.6 \pm 2.5$ & $10.2 \pm 2.1$ & $9.9 \pm 1.1$ \\
\hline $\mathrm{JAM}^{3}$ & $12.7 \pm 1.4$ & $8.2^{b} \pm 0.5$ & $9.4 \pm 0.7$ & $7.1 \pm 1.3$ & $2.4 \pm 0.6$ & $13.4 \pm 2.5$ & $8.8 \pm 2.1$ & $9.7 \pm 1.1$ \\
\hline \multicolumn{9}{|c|}{ L-lactate } \\
\hline $\mathrm{CON}^{2}$ & $17.2 \pm 7.9$ & $19.2 \pm 6.9$ & $37.2 \pm 19.3$ & $4.1 \pm 4.2$ & $0.0 \pm 0.0$ & $0.0^{\mathrm{C}} \pm 0.0$ & $0.1 \pm 0.0$ & $0.2 \pm 0.1$ \\
\hline $\mathrm{JAM}^{3}$ & $34.9 \pm 9.5$ & $24.9 \pm 6.9$ & $13.5 \pm 15.7$ & $10.2 \pm 3.9$ & $0.0 \pm 0.0$ & $0.0^{\mathrm{d}} \pm 0.0$ & $0.0 \pm 0.0$ & $0.2 \pm 0.1$ \\
\hline \multicolumn{9}{|c|}{ D-lactate } \\
\hline $\mathrm{CON}^{2}$ & $19.7 \pm 6.9$ & $8.8 \pm 6.9$ & $22.6 \pm 11.6$ & $4.0 \pm 7.2$ & $0.1 \pm 0.0$ & $0.1 \pm 0.0$ & $0.1 \pm 0.0$ & $0.3 \pm 0.1$ \\
\hline $\mathrm{JAM}^{3}$ & $23.6 \pm 6.9$ & $27.7 \pm 6.9$ & $15.9 \pm 9.5$ & $16.0 \pm 6.9$ & $0.0 \pm 0.0$ & $0.0 \pm 0.0$ & $0.1 \pm 0.0$ & $0.2 \pm 0.1$ \\
\hline \multicolumn{9}{|c|}{ 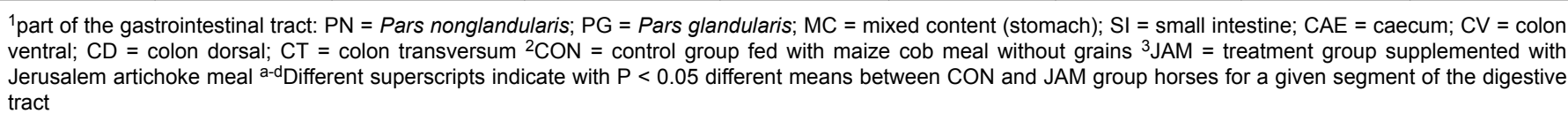 } \\
\hline
\end{tabular}




\section{Ammonia}

Feeding of JAM elevated the ammonia concentration predominantly in the stomach (PN, PG and $\mathrm{MC}$ ) as well as in the ventral colon (Table 3). Particularly in the Pars glandularis, the concentration of ammonia was significantly higher in the JAM group in comparison to the control group (Table 3; $\mathrm{P}<$ $0.05)$. The concentration of ammonia was tendentially lower in the small intestine, caecum, colon dorsale and colon transversum when JAM was fed (Table $3 ; \mathrm{P}>0.05$ ).

\section{Discussion}

Feed analysis indicate that the horsed received $199 \mathrm{~g}$ of mono- and dimeric sugars but only $466 \mathrm{~g}$ of fructans via $1 \mathrm{~kg}$ of the commercial JAM product (as fed basis) being $161 \mathrm{~g} / \mathrm{kg}$ lower than declared. From that, the horses did not have ingested as much prebiotic active substances as it is recommended to be prebiotic [8] and thus was aimed in the study. On the one hand, this has probably influenced the outcome of the study. On the other hand, differences between assumed and realized contents of inulin-type fructans in the additive in question may have biased former studies too. The authors of the Present study are not aware of analytical control of the fructan content in the majority of studies from the literature. To overcome the problem analytical control might further be an issue for both science and practical use. The amount of starch intake was calculated based on feedstuff analysis before the study. During the experiment, the pooled sample (oat grains) was analyzed once again and a higher starch content was measured. Therefore, the starch intake was $1.2 \mathrm{~g} / \mathrm{kg} \mathrm{BW}^{*} \mathrm{~d}^{-1}$ instead of exactly $1 \mathrm{~g}$ starch per kg BW and day.

Nevertheless, the horses consumed the moistened concentrates quite well during the entire study. The stomach was full of digesta after euthanasia and the stomachs' DM content measured in the recent study is in the range of what is reported from the literature [26]. Despite no significant differences were noticed the DM content in nearly all parts of the gastrointestinal tract was numerically higher when JAM was fed which coincided with also higher total concentrations of SCFA. Elevated SCFA might have triggered water absorption probably at least in the terminal gut. An argument against this explanation is the DM content in the colon transversum being just there higher in CON vs. JAM horses.

Feeding of prebiotics aimed to elevate the microbial activity associated with an increase of microbial fermentation products predominantly in the hindgut [12]. Contrary, the results of the present study indicate that an elevated microbial metabolism already exists in the foregut following JAM intake. Feeding of JAM increased the total amounts of SCFA in both parts of the stomach. Interestingly, the percentages of acetic acid of total SCFA were almost similar in both parts of the stomach (Pars nonglandularis and Pars glandularis) after feeding of JAM, but both the total amount and the percentage of $n$-butyric acid as well tended to increase with coincident reduction of the percentage of propionic acid. $\mathrm{N}$-butyric acid was reported to have several beneficial impacts on the gut epithelium e.g. energy source for the colonic epithelial cells, activation of mucin release, regulation of cell proliferation and apoptosis [14] primarily in the hindgut. In the equine large intestine, butyrate transport can be mediated by the monocarboxylate transporter 1 (MCT 1) and inhibited through luminal acetate, propionate and lactate concentrations of about $20 \mathrm{mmol} / \mathrm{L}$ [27]. As opposed to this, the impact of $n$ butyric acid in the stomach seems to be detrimental. In an in vitro study with tissues from the nonglandular part of the equine stomach, $n$-butyric acid significantly decreased the mucosal barrier function at a concentration of $60 \mathrm{mmol} / \mathrm{L}$ and in combination with a low pH of 4.0 or even 1.5 [28]. At least $\mathrm{pH}$ values around 4 are normally reached in the stomach content of horses even rather on hay than concentrate-based diets [26] as it was provided here. The $\mathrm{pH}$ levels measured in the recent study were actually between 4.4 and 3.4 in the nonglandular and glandular region, respectively, of the stomach. Therefore, higher amounts of $n$-butyric acid together with a low $\mathrm{pH}$ might have been an issue in the pathogenesis of gastric ulcers related to the nonglandular part of the equine stomach. The horses in the recent study did however not exhibit gastrointestinal disturbances or any other health discomfort during the study period. Post mortem observation of the stomach wall, however, did also not indicate that 3 weeks feeding of JAM in a dose chosen here causes mucosal lesions in any part of the organ. The only horse with mucosal lesions in the stomach belonged to the control group. It needs, however, be taken into account that the measurements were performed in digesta sampled approximately 1 hour after concentrate intake. This means that the availability of rapidly fermentable carbohydrates was rather high at this time point, and thus the concentration of organic acids might be as well. The subsequent time course over the day has probable mitigate this. It is further imaginable that the 3 weeks lasting adaptation period was not long enough for a clear impact of the diet on the gastric mucosa health. The higher concentrations of $n$-butyric acid in the stomach might be a result of two potential mechanisms. Firstly, the supplementation of prebiotic compounds can have increased the amount of butyric acid producing bacteria like Sarcina spp. or Clostridium spp. [29]. Consequently, the elevated microbial metabolism due to the increased availability of specific substrates would lead to an overall increased content of fermentation products, particularly $n$-butyric acid. Secondly, the increased concentration of this particular SCFA might be a result of interconversion reactions. Results from an in vitro study revealed that there is a considerable contribution of interconversion reactions from extracellular acetate as well as lactate to butyrate after adding oligo-fructose as substrate [30]. The present results indicate a tendency for higher contents of L-lactate as well as of D-lactate especially in both parts of the stomach. There was also a tendency for higher contents of $n$-butyric acid which was notably noticed in the Pars nonglandularis, but only a slight increase in the Pars glandularis. The dominant bacteria in the horse stomach were found to be Lactobacilli spp., Sarcina spp. and Streptococci spp. [29]. Consequently, the elevated metabolism of the dominant Lactobacilli spp. might result in a higher concentration of lactate in the stomach, particularly in the Pars nonglandularis. 
This might enhance other bacteria (like Sarcina spp.) to convert lactate to $n$-butyric acid leading to an overall increased amount of this intrinsic SCFA.

Moreover, the results indicated a tendential increase of the content of iso-valeric acid in the Pars glandularis and only a slight increase in the Pars nonglandularis. In vitro studies with nonglandular tissue samples revealed a decrease in the barrier function after the incubation with iso-valeric acid $(60 \mathrm{mmol} / \mathrm{L})$ at $\mathrm{pH} \leq 7.0$ [31]. Iso-types of SCFA indicate microbial proteolysis which is in good increment with the higher ammonia contents in the stomach digesta following JAM feeding in this study. Further, branched-chained SCFA are not rapidly absorbable in the nonglandular part of the stomach [32], which might result in a further accumulation in the glandular part of this organ.

Prebiotics are used to stimulate predominantly the autochthonous microflora as well as their metabolism in the hindgut [12]. In contrast to studies with scFOS fed in different dosages $[10,11]$, the present results indicated a much lesser impact of JAM on the microbial fermentation products in the hindgut than expected. Already in a former study fermentation gases in the exhaled air indicated that inulin started to be fermented in more distal parts of the horses' gut [13]. In the current study, feeding of JAM was accompanied by lower concentrations of SCFA and lactate (L- and D- isomer) in the caecum. An increased content of L-lactate was noticed after feeding of scFOS (30 g/d $\times$ horse ${ }^{-1}$, over a $21 \mathrm{~d}$ period), but there was no impact on the concentration of total SCFA [11]. In the ventral colon, the content of $n$-butyric acid as well as acetic acid were elevated and the concentration of L-lactate was significantly reduced in the recent study which needs to be assessed rather fortunately in this part of the digestive tract. In contrast to this, the supplementation of scFOS had no impact on the concentration of SCFA and decreased the amount of D-lactate but not L-lactate in the colon. In the study of Respondek et al. [11], horses (7 years old) supplemented with scFOS were fed at a roughage to concentrate ratio of approximately 30:70. The horses in the current study received a vice versa ratio of approximately 85:15. Therefore, apart from the dosage of the prebiotic, the composition of the basal ration might be relevant for the effects of the prebiotic especially in the hindgut. Diets containing more than $50 \%$ of concentrate can lead to an augmented concentration of SCFA in the hindgut, so that the prebiotic effect might be less pronounced [33].

Furthermore, feeding of JAM resulted in a tendential increased concentration of ammonia in the Pars nonglandularis and a significantly elevated content in the Pars glandularis. This might be a result of an increased metabolic activity of several proteolytic and/or ureolytic microorganisms in the stomach as mentioned above. Some studies indicated the highest proteolytic activity primarily in the small intestine of horses [34] but more recent results revealed the presence of proteolytic active bacteria like Pseudomonas spp. equally in the stomach [30]. Due to the low pH in the stomach, urease seems to be inactive but the increased amount of ammonia might be a result of the contribution of intracellular gastric urease originated from bacteria $[35,36]$. Elevated amounts of ammonia were also noticed in the hindgut particularly in the ventral colon after feeding of JAM. Redundant amounts of ammonia could either be excreted via feces or be absorbed and converted to non-proteinogenic amino acids or urea in the liver $[35,37]$.

Although the supplementation of JAM increased the concentration of SCFA and lactate predominantly in the stomach, no $\mathrm{pH}$ differences between both groups were recorded. This might be based upon the somewhat higher elevated concentration of ammonia in the stomach following JAM feeding, which acts as puffer therefore counteracts a $\mathrm{pH}$ decrease. Particularly in human being and may be other target animals such as pigs feeding of prebiotics aims, among others, to decrease the $\mathrm{pH}$ predominantly in the hindgut and thus to reduce the chance of pathogens to colonize the digestive tract [9]. The present results indicated no decreasing $\mathrm{pH}$ in the hindgut related to only a slight increase of organic acids as acidifying fermentation products and in some anatomical regions a concomitant ammonia increase.

In the recent study, the amount of prebiotics that actually might have reached the hindgut seemed to be much lesser than required and did not lead to a significant elevation of the microbial activity. An elevation of the dose, however, may increase the risk for pronounced microbial fermentation although in the stomach with subsequent negative impact on the mucosal health and can therefore not unconditionally be recommended. This issue needs to be investigated further as well as probable impacts of the chain length of individual fructans in the prebiotic in question, which are partly responsible for the rapidness of degradation. And last but not least, future galenic treatment might ensure prebiotics to be fermented first in the hindgut where they actually should be decomposed to unfold their positive effects. When working on such a strategy one should consider that apparently prebiotic doses recommended for practical use [8] unavoidably included that part of prebiotic substances that have been decomposed by both microbial fermentation and acidic hydrolysis particularly in the stomach of horses. Thus, the situation when the full prebiotic dose as recommended by [8] would actually reach the caecum needs to be investigated carefully.

\section{Conclusions}

Fructo-oligosaccharides + inulin originating from Jerusalem artichoke meal fed to horses in an apparently prebiotic dosage might cause an increased microbial activity and equally elevated amounts of fermentation products predominantly in the stomach. Although increased acidity represents a risk factor for gastric ulcers this effect needs to be discussed carefully with respect to particular SCFA, the elevated ammonia concentration and the fairly stable $\mathrm{pH}$ value as overall result. The impact of feeding the prebiotic compound in the dosage applied here on the hindgut might be much lesser pronounced than expected. 


\section{Acknowledgement}

The authors would like to acknowledge the technical staff of the University of Veterinary Medicine in Hannover for animal care and feeding as well as for laboratory assistance. Furthermore, the authors express their gratitude for excellent laboratory assistance to the technical staff of the Martin Luther University, Group Animal Nutrition, especially to D. Schmiedel, K. Sitte and A. Schmidt.

\section{References}

1. De Fombelle A, Julliand V, Drogoul C, Jacotot E (2001) Feeding and microbial disorders in horses: 1-effects of an abrupt incorporation of two levels of barley in a hay diet on microbial profile and activities. Journal of Equine Veterinary Science 21 439-445.

2. Longland AC, Byrd BM (2006) Pasture nonstructural carbohydrates and equine laminitis. Journal of Nutrition 136: 2099S-2102.

3. Milinovich GJ, Trott DJ, Burrell PC, van Eps AW, Thoefner MB, et al. (2006) Changes in equine hindgut bacterial populations during oligofructose-induced laminitis. Environmental Microbiology 8: 885-898.

4. Muhonen S, Julliand V, Lindberg JE, Bertilsson J, Jansson A, et al. (2009) Effects on the equine colon ecosystem of grass silage and haylage diets after an abrupt change from hay. Journal of Animal Science 87: 2291-2298.

5. Van Eps AW, Pollit CC (2006) Equine laminitis induced with oligofructose. Equine Veterinary Journal 38: 203-208.

6. Van Eps AW, Pollit CC (2009) Equine laminitis model: Lamellar histopathology seven days after induction with oligofructose. Equine Veterinary Journal 41: 735-740.

7. Respondek F, Goachet AG, Rudeaux F, Julliand V (2007) Effects of short-chain fructo-oligosaccharides on the microbial and biochemical profile of different segments of the gastrointestinal tract in horses. Pferdeheilkunde 23: 146-150.

8. Julliand V, Zeyner A (2013) Dietary strategies for optimizing gastro intestinal health: an update on pre- and probiotics. In: Horse Health Nutrition, European Equine Health \& Nutrition Congress, pp: 75-84.

9. Van Loo J (2004) The specificity of the interaction with intestinal bacterial fermentation by prebiotics determines their physiological efficacy. Nutrition Research Reviews 17: 89-98.

10. Berg EL, Fu CJ, Porter JH, Kerley MS (2005) Fructooligosaccharide supplementation in the yearling horse: Effects on fecal $\mathrm{pH}$, microbial content and volatile fatty acid concentration. Journal of Animal Science 83: 1549-1553.

11. Respondek F, Goachet AG, Julliand V (2008) Effects of dietary short-chain fructooligosaccharides on the intestinal microflora of horses subjected to a sudden change in diet. Journal of Animal Science 86: 316-323.

12. Samanta AK, Jayapal N, Senani S, Kolte AP, Sridhar M, et al. (2013) Prebiotic inulin: Useful dietary adjuncts to manipulate the livestock gut microflora. Sociedade Brasileira de Microbiologia 44: 1-14.

13. Coenen M, Mößeler A, Vervuert I (2006) Fermentative gases in breath indicate the Inulin and starch start to be degraded by microbial fermentation in the stomach and small intestine of the horse in contrast to Pectin and Cellulose. The Journal of Nutrition 136: 2108S-2110.

14. Plöger S, Stumpff F, Penner GB, Schulzke JD, Gäbel G, et al. (2012) Microbial butyrate and its role for barrier function in the gastrointestinal tract. Annals of the New York Academy of Sciences 1258: 52-59.

15. Energy and nutrient requirements of farm animals. (2014) No. 11 Recommendations for energy and nutrients of horses (Society for Nutritional Physiology). DLG -Verlag, Frankfurt.

16. Pavis N, Chatterton NJ, Harrison PA, Baumgartner S, Praznik, et al. (2001) Structure of fructans in roots and leaf issues of Lolium perenne. New physiologist 150: 83-95.

17. Association of German agricultural study and research institutes (VDLUFA) : Handbook of the Agricultural Experimental and research methodology (VDLUFA Methodenbuch). Volume III : The chemical control of feed (supplementary deliveries), VDLUFA Verlag, Darmstadt, 2015.

18. Zeyner A, Gefrom A, Hillegeist D, Sommer M, Greef JM, et al. (2015) Contribution to the method of sugar analysis in legume grains for ensiling- A pilot study. International Journal of Scientific Research in Science and Technology 1: 1-22.

19. Zeyner A, Geißler C, Dittrich A (2004) Effects of hay intake and feeding sequence on variables in faeces and faecal water (dry matter, pH value, organic acids, ammonia, buffering capacity) of horses. Journal of Animal Physiology and Animal Nutrition 88: 7-19.

20. Scheijen JLJM, Hanssen NMJ, van de Waarenburg MPH, Jonkers DMAE, Stehouwer CDA, et al. (2012) L(+) and D(-) lactate are increased in plasma and urine samples of type 2 diabetes as measured by a simultaneous quantification of $L(+)$ and $D(-)$ lactate by reversed-phase liquid chromatography tandem mass spectrometry. Experimental diabetes research.

21. Henry H, Marmy Conus N, Steenhout P, Béguin A, Boulat O, et al. (2012) Sensitive determination of D-lactic acid and L-lactic acid in urine by high-performance liquid chromatography-tandem mass spectrometry. BMC 26: 425-428.

22. Conway EJ, Byrne A (1933) An absorption apparatus for the micro-determination of certain volatile substances: the microdetermination of ammonia. Biochemical Journal 27: 419-429.

23. Voigt J, Steger H (1967) For the quantitative determination of ammonia, urea and ketone bodies in biological materials using a modified micro-diffusion vessel. Archives of Animal Nutrition 17: 289-293.

24. Kienzle E, Zeyner A (2010) The development of a metabolizable energy system for horses. Journal of Animal Physiology and Animal Nutrition 94: e231-e240.

25. Zeyner A, Kirchhof S, Susenbeth A, Südekum KH, Kienzle E, et al. (2015) A new protein evaluation system for horse feed from literature data. Journal of Nutritional Sciences.

26. Coenen M (1992) Observations on the occurrence of gastric ulcers in horses. Pferdeheilkunde, pp: 188-191.

27. Nedjadi T, Moran AW, Al-Rammahi MA, Shirazi-Beechey SP (2014) Characterization of butyrate transport across the luminal membranes of equine large intestine. Experimental Physiology 99: 1335-1347.

28. Nadeau JA, Andrews FM, Patton CS, Argenzio RA, Mathew AG, et al. (2003) Effects of hydrochloric, acetic, butyric, and propionic acids on pathogenesis of ulcers in the nonglandular portion of 
the stomach of horses. American Journal of Veterinary Research 64: 404-412.

29. Costa MC, Silva G, Ramos RV, Staempfli HR, Arroyo LG, et al. (2015) Characterization and comparison of the bacterial microbiota in different gastrointestinal tract compartments in horses. Veterinary journal 205: 74-80.

30. Morrison DJ, Mackay WG, Edwards CA, Preston T, Dodson B, et al. (2006) Butyrate production from oligofructose fermentation by the human faecal flora: what is the contribution of extracellular acetate and lactate? British Journal of Nutrition 96 : 570-577.

31. Nadeau JA, Andrews FM, Patton CS, Argenzio RA, Mathew AG, et al. (2003) Effects of hydrochloric, valeric and other volatile fatty acids on pathogenesis of ulcers in the nonglandular portion of the stomach of horses. American Journal of Veterinary Research 64: 413-417.

32. Nadeau JA, Andrews FM, Mathew AG, Argenzio RA, Blackford JT, et al. (2000) Evaluation of diet as cause of gastric ulcers in horses. American Journal of Veterinary Research 61: 784-790.
33. Julliand V, Fombelle A de, Drogoul C, Jacotot E (2001) Feeding and microbial disorders in horses: Part 3-Effects of three hay: grain ratios on microbial profile and activities. Journal of Equine Veterinary Science 21: 543-546.

34. Kern DL, Slyter LL, Leffel EC, Weaver JM, Oltjen RR, et al. (1974) Ponies vs. Steers: Microbial and chemical characteristics of intestinal. Journal of Animal Science 38: 559-564.

35. Hecker JF (1971) Ammonia in the large intestine of herbivores. British Journal of Nutrition 26: 135.

36. Kornberg HL, Davies RE (1955) Gastric urease. Physiological Reviews 35: 169-177.

37. Martin RG, McMeniman NP, Norton BW, Dowsett KF (1996) Utilization of endogenous and dietary urea in the large intestine of the mature horse. British Journal of Nutrition 76: 373-386. 